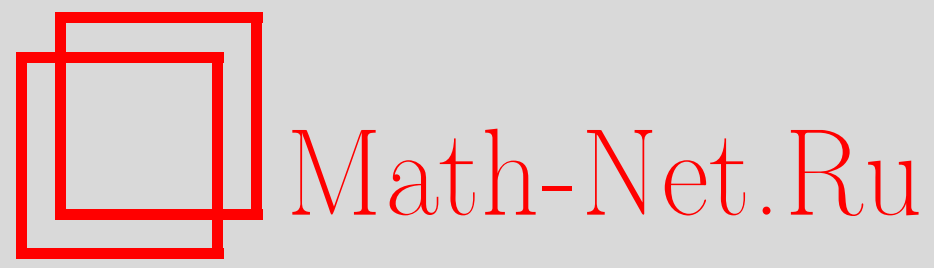

И. А. Круглов, Условия предельной равновероятности состояний регистров сдвига, Матем. вопр. криптогр., 2010, том 1, выпуск 2, 19-29

DOI: https://doi.org/10.4213/mvk8

Использование Общероссийского математического портала Math-Net.Ru подразумевает, что вы прочитали и согласны с пользовательским соглашением

http://www.mathnet.ru/rus/agreement

Параметры загрузки:

IP : 107.22 .136 .117

26 апреля 2023 г., 11:53:31 
УДК: 512.542, 519.217.2

\title{
Условия предельной равновероятности состояний регистров сдвига
}

\author{
И. А. Круглов \\ ООО «Центр сертификационных исследований», Москва
}

Получено 22.IV.2010

В работе описаны условия предельной равновероятности состояний регулярных регистров сдвига в случае, когда на вход регистра поступает последовательность случайных входных символов, связанных марковской зависимостью.

Ключевые слова: регистр сдвига, равномерное распределение, цепь Маркова

Conditions of limit uniformity of shift register state distribution

\section{A. Kruglov}

LLC "Certification Research Center", Moscow

Abstract. A shift register with Markovian input sequence is considered. Sufficient conditions for the convergence of register state distribution to the uniform law are given. Key words: shift register, uniform distribution, Markov chain

Citation: Mathematical Aspects of Cryptography, 2010, vol. 1, no. 2, pp. 19-29 (Russian). 
Пусть

$$
\alpha_{1}, \alpha_{2}, \ldots, \alpha_{N}, \ldots
$$

- конечная простая однородная неразложимая цепь Маркова с множеством состояний $\{1,2, \ldots, n\}$, матрицей переходных вероятностей $P=\|p(i, j)\|$ и некоторым положительным вектором начального распределения, $(G ; \cdot)-$ конечная группа. Предположим, что для любых $i, j \in\{1,2, \ldots, n\}$ задана последовательность $\left\{\xi_{i, j}^{(N)}\right\}$ одинаково распределенных со случайной величиной $\xi_{i, j}$ случайных величин со значениями в группе $G$. Пусть группа

$$
G^{(m)}=\underbrace{G \times G \times \ldots \times G}_{m}, \quad m \geq 1,
$$

есть $m$-я декартова степень группы $G,\left(\zeta_{1}, \ldots, \zeta_{m}\right)-$ случайный вектор со значениями в группе $G^{(m)}$. Будем предполагать, что случайные величины $\left(\zeta_{1}, \ldots, \zeta_{m}\right), \xi_{i, j}^{(N)}(N \geq 1, i, j \in\{1, \ldots, n\})$ и цепь Маркова (1) независимы в совокупности.

Предположим, что задано отображение

$$
\varphi: G^{(m)} \rightarrow G
$$

удовлетворяющее следующему условию регулярности: для любых $g_{1}, g_{2}, \ldots, g_{m-1} \in G$ биективно отображение

$$
\varphi_{g_{1}, \ldots, g_{m-1}}: G \rightarrow G
$$

где $\varphi_{g_{1}, \ldots, g_{m-1}}(g)=\varphi\left(g, g_{1}, \ldots, g_{m-1}\right), g \in G$.

Определим случайные величины со значениями в группе $G$ :

$$
\zeta_{m+N}=\varphi\left(\zeta_{N}, \zeta_{N+1}, \ldots, \zeta_{N+m-1}\right) \cdot \xi_{\alpha_{N}, \alpha_{N+1}}^{(N)}, N \geq 1,
$$

а также случайные величины со значениями в группе $G^{(m)}$ :

$$
\zeta^{(0)}=\left(\zeta_{1}, \ldots, \zeta_{m}\right), \quad \zeta^{(N)}=\left(\zeta_{N+1}, \zeta_{N+2}, \ldots, \zeta_{N+m}\right), N \geq 1 .
$$

Ранее последовательность (3) в случае $n=1$ изучалась в работах $[3,4,6]$. Последовательность (3) описывает вероятностные свойства последовательности состояний регистра сдвига с функцией обратной связи $\varphi$ при наличии случайной марковской входной последовательности.

Обозначим через $p^{(N)}(i, j)$ вероятность перехода цепи Маркова (1) из состояния $i$ в состояние $j$ за $N$ шагов. Для таких $N, i, j$, что $p^{(N)}(i, j)>0$, 
рассмотрим случайные величины $\zeta_{i, j}^{(N)}$ со значениями в группе $G^{(m)}$ : распределение $\zeta_{i, j}^{(N)}$ есть условное распределение случайной величины $\zeta^{(N)}$ при условии $\alpha_{1}=i, \alpha_{N+1}=j$.

В данной работе мы исследуем условия предельной при $N \rightarrow \infty$ равномерности на группе $G^{(m)}$ распределений последовательности случайных величин (3), а также последовательностей случайных величин

$$
\left\{\zeta_{i, j}^{(N)}\right\}, i, j \in\{1, \ldots, n\}
$$

Для случайной величины $\xi$ со значениями в конечной группе будем обозначать через $\Omega(\xi)$ носитель ее распределения.

Теорема 1. Пусть выполнено условие

$$
\bigcap_{\substack{j \in 1, n: \\ p(i, j)>0}} \Omega\left(\xi_{i, j}\right) \neq \oslash, i \in\{1, \ldots, n\},
$$

неотрицательная матрииа $P^{\prime} \cdot P$ неразложима $\left({ }^{\prime}\right.$ - знак транспонирования), и не существует собственной подгруппь $L$ группы $G$, для которой множество

$$
\Omega=\bigcup_{\substack{i, j \in \overline{1, n} \\ p(i, j)>0}} \Omega\left(\xi_{i, j}\right)
$$

содержится в одном смежном классе группь $G$ по подгруппе L. Тогда для любого регулярного отображения $\varphi$, любого распределения случайной величины $\left(\zeta_{1}, \zeta_{2}, \ldots, \zeta_{m}\right)$ и любых $i, j \in\{1, \ldots, n\}$ последовательность распределений случайных величин (4) имеет своим пределом при $N \rightarrow \infty$ равномерное распределение на множестве $G^{(m)}$.

Доказательство. Рассмотрим симметрическую группу подстановок $S\left(G^{(m)}\right)$ множества $G^{(m)}$ с операцией произведения отображений. Каждому элементу $g \in G$ поставим в соответствие элемент $\pi(g) \in S\left(G^{(m)}\right)$ следующим образом:

$$
\pi(g)\left(\left(g_{1}, \ldots, g_{m}\right)\right)=\left(g_{2}, \ldots, g_{m}, \varphi\left(g_{1}, \ldots, g_{m}\right) \cdot g\right), \quad\left(g_{1}, \ldots, g_{m}\right) \in G^{(m)} .
$$

Множество $\{\pi(g) \mid g \quad \in G\}$ порождает некоторую группу подстановок множества $G^{(m)}$, которую мы будем обозначать через $\left(\mathfrak{S}^{*}, *\right)$. 
Каждой случайной величине $\xi$ со значениями в группе $G$ соответствует случайная величина со значениями в группе $\mathfrak{O})$, которую мы будем обозначать $\widetilde{\xi}$, значения $\widetilde{\xi}$ определяются равенством $\widetilde{\xi}=\pi(\xi)$. Заметим, что при этом распределения $\xi$ и $\widetilde{\xi}$ связаны равенством

$$
\boldsymbol{P}(\xi=g)=\boldsymbol{P}(\widetilde{\xi}=\pi(g)), g \in G
$$

и что из независимости случайных величин со значениями в группе $G$ из некоторого семейства $\left\{\xi_{\beta}\right\}$ следует независимость случайных величин со значениями в группе $\mathfrak{S}$ из соответствующего семейства $\left\{\widetilde{\xi}_{\beta}\right\}$.

Введем в рассмотрение вспомогательные случайные величины. Пусть

$$
\theta_{1}, \theta_{2}, \ldots, \theta_{N}, \ldots
$$

- последовательность взаимно независимых и не зависящих от $\zeta^{(0)}$ случайных величин со значениями в группе $G$, имеющих равномерное распределение на множестве $\Omega$ (см. (6)). Определим следующие случайные величины со значениями в группе $G$ :

$$
\begin{gathered}
\mu_{N}=\zeta_{N}, N \in\{1, \ldots, m\}, \\
\mu_{m+N}=\varphi\left(\mu_{N}, \ldots, \mu_{N+m-1}\right) \cdot \theta_{N}, N \geq 1 .
\end{gathered}
$$

Далее введем случайные величины со значениями в группе $\mathfrak{( 5 :}$

$$
\begin{gathered}
\widetilde{\theta}^{(N)}=\widetilde{\theta}_{1} * \widetilde{\theta}_{2} * \ldots * \widetilde{\theta}_{N}, N \geq 1, \\
\widetilde{\eta}^{(N)}=\widetilde{\xi}_{\alpha_{1}, \alpha_{2}}^{(1)} * \widetilde{\xi}_{\alpha_{2}, \alpha_{3}}^{(2)} * \ldots * \widetilde{\xi}_{\alpha_{N}, \alpha_{N+1}}^{(N)}, N \geq 1 .
\end{gathered}
$$

Для каждых $i, j \in\{1, \ldots, n\}, N \geq 1$, удовлетворяющих условию $p^{(N)}(i, j)>0$, рассмотрим случайную величину $\widetilde{\eta}_{i, j}^{(N)}$ со значениями в $\mathfrak{G}$, распределение которой совпадает с условным распределением $\widetilde{\eta}^{(N)}$ при условии $\alpha_{1}=i, \alpha_{N+1}=j$.

Мы будем также рассматривать случайные величины

$$
\mu^{(N)}=\left(\mu_{N+1}, \mu_{N+2}, \ldots, \mu_{N+m}\right), N \geq 0,
$$

со значениями в группе $G^{(m)}$.

Будем считать, что зафиксирована некоторая нумерация элементов группы $G^{(m)}$, и в соответствии с этой нумерацией определяются векторы $\bar{p}_{\eta}$ распределений случайных величин $\eta$ со значениями в группе $G^{(m)}$, а также 
матрицы переходных вероятностей $\prod_{\widetilde{\xi}}$ случайных величин $\widetilde{\xi}$ со значениями в группе $\mathfrak{G}$, см. [2]. Справедливы следующие равенства:

$$
\begin{gathered}
\bar{p}_{\zeta_{i, j}^{(N)}}=\bar{p}_{\zeta^{(0)}} \cdot \prod_{\widetilde{\eta}_{i, j}^{(N)}}, i, j \in\{1, \ldots, n\}, N \geq 1, p^{(N)}(i, j)>0, \\
\bar{p}_{\mu^{(N)}}=\bar{p}_{\zeta^{(0)}} \cdot \prod_{\widetilde{\theta}^{(N)}}, N \geq 1 .
\end{gathered}
$$

Равенство (8) следует из соотношения

$$
P\left(\zeta^{(N)}=\bar{h}\right)=\sum_{\bar{g} \in G^{(m)}} P\left(\zeta^{(0)}=\bar{g}\right) \cdot \sum_{\substack{\tau \in(\mathcal{G}: \\ q(\tilde{\tau})(\bar{g})=\bar{h}}} P\left(\widetilde{\eta}_{i, j}^{(N)}=\widetilde{\tau}\right)
$$

и из того факта, что для любых $\bar{h}, \bar{g} \in G^{(m)}$ число

$$
\sum_{\substack{\tau \in(\mathfrak{G}) \\ \tau(\bar{g})=\bar{h}}} \boldsymbol{P}\left(\widetilde{\eta}_{i, j}^{(N)}=\widetilde{\tau}\right)
$$

есть элемент матрицы $\prod_{\widehat{\eta}_{i, j}^{(N)}}$ в строке с номером $\bar{g}$ и столбце с номером $\bar{h}$. Равенство (9) доказывается аналогично.

В соответствии с утверждением следствия 2 в работе [5] для любых $i, j \in\{1, \ldots, n\}$ и любой такой возрастающей последовательности $\left\{k_{N}\right\}$, натуральных чисел, что $p^{\left(k_{N}\right)}(i, j)>0$ при всех $N \geq 1$, последовательности распределений случайных величин

$$
\left\{\bar{p}_{\widetilde{\eta}_{i, j}^{\left(k_{N}\right)}}\right\},\left\{\bar{p}_{\widetilde{\theta}^{\left(k_{N}\right)}}\right\}
$$

сходятся или не сходятся одновременно, и в случае сходимости их пределы равны.

Пусть $\left\{k_{N}\right\}-$ произвольная возрастающая последовательность натуральных чисел, для которой последовательность распределений $\left\{\bar{p}_{\zeta_{i, j}^{\left(k_{N}\right)}}\right\}$ имеет предел. По теореме Больцано - Вейерштрасса из последовательности распределений случайных величин $\left\{\bar{p}_{\widetilde{\theta}^{\left(k_{N}\right)}}\right\}$ можно выбрать сходящуюся подпоследовательность $\left\{\bar{p}_{\widetilde{\theta}^{\left(k_{s(N)}\right)}}\right\}$. По теореме о выражении матриц переходных вероятностей случайных величин со значениями в конечных группах подстановок через их распределения, см. [2], последовательности матриц переходных вероятностей

$$
\left\{\prod_{\widetilde{\eta}_{i, j}^{\left(k_{s(N)}\right)}}\right\},\left\{\prod_{\tilde{\theta}_{i, j}^{\left(k_{s(N)}\right)}}\right\}
$$

2010, T. 1, № 2, C. 19-29 
сходятся при $N \rightarrow \infty$, и их пределы равны. Из соотношений (8), (9) следует, что для любых $i, j \in\{1, \ldots, n\}$ последовательности распределений

$$
\left\{\bar{p}_{\zeta_{i, j}^{\left(k_{s(N)}\right)}}\right\},\left\{\bar{p}_{\mu^{\left(k_{s(N)}\right)}}\right\}
$$

сходятся при $N \rightarrow \infty$, и их пределы равны. Из соотношения (7), условия теоремы на носитель $\Omega$ распределений случайных величин $\theta_{N}, N \geq 1$, и теоремы 2 работы [4] следует, что последовательность распределений $\left\{\bar{p}_{\mu^{\left(k_{s(N)}\right)}}\right\}$ сходится при $N \rightarrow \infty$ к равномерному распределению на группе $G^{(m)}$. Таким образом последовательность $\left\{\bar{p}_{\zeta_{i, j}^{\left(k_{s(N)}\right)}}\right\}$, а с ней и последовательность $\left\{\bar{p}_{\zeta_{i, j}^{\left(k_{N}\right)}}\right\}$, при $N \rightarrow \infty$ имеет своим пределом равномерное распределение на группе $G^{(m)}$. Так как каждая сходящаяся подпоследовательность последовательности $\left\{\bar{p}_{\zeta_{i, j}^{(N)}}\right\}$ сходится к равномерному распределению на группе $G^{(m)}$, то и последовательность $\left\{\bar{p}_{\zeta_{i, j}^{(N)}}\right\}$ при $N \rightarrow \infty$ имеет своим пределом равномерное распределение на группе $G^{(m)}$.

Теорема 1 доказана.

Заметим, что условие неразложимости матриц $P$ и $P^{\prime} \cdot P$ равносильно известному условию А. Н. Колмогорова (см. [7]) на цепь Маркова (1).

Используя формулу полной вероятности и теорему 1 , можно доказать следующее утверждение.

Следствие 1. Пусть выполнены условия теоремы 1, тогда для любого регулярного отображения $\varphi$, любого распределения случайной величины $\left(\zeta_{1}, \zeta_{2}, \ldots, \zeta_{m}\right)$ последовательность распределений случайных величин (3) имеет своим пределом при $N \rightarrow \infty$ равномерное распределение на груnпе $G^{(m)}$.

Рассмотрим следующий пример.

Опишем цепь Маркова (1). Пусть $k \geq 1$, и для каждого $j \in\{1, \ldots, k\}$ задана последовательность случайных величин $\left\{x_{N}^{(j)}\right\}$, связанная в некоторую положительно-регулярную цепь Маркова с множеством состояний $\{0 ; 1\}$, некоторой положительно-регулярной матрицей переходных вероятностей $P_{j}$ и некоторым положительным вектором начального распределения. Будем предполагать, что цепи Маркова $\left\{x_{N}^{(j)}\right\}, j \in\{1, \ldots, k\}$, независимы в совокупности. В качестве цепи Маркова (1) рассмотрим последовательность векторов

$$
\left\{\left(x_{N}^{(1)}, x_{N}^{(2)}, \ldots, x_{N}^{(k)}\right)\right\} .
$$


Таким образом, в данном случае множество состояний цепи Маркова (1) есть множество $V_{k}$ всех двоичных векторов длины $k$, число состояний $n=2^{k}$ и матрица $P$ переходных вероятностей цепи Маркова (1) есть тензорное произведение матриц

$$
P=P_{1} \otimes P_{2} \otimes \ldots \otimes P_{k}
$$

Наконец, для произвольной пары векторов $i=\left(i_{1}, \ldots, i_{k}\right), j=\left(j_{1}, \ldots, j_{k}\right) \in V_{k}$ и любого $N \geq 1$ будем считать, что распределение случайной величины $\xi_{i, j}^{(N)}$ есть вырожденное распределение в элементе $f\left(i_{1}, \ldots, i_{k}\right) \in G$, где $f$ - некоторая псевдобулева функция от $k$ переменных. В этом случае соотношение (2) принимает следующий вид:

$$
\zeta_{m+N}=\varphi\left(\zeta_{N}, \zeta_{N+1}, \ldots, \zeta_{N+m-1}\right) \cdot f\left(x_{N}^{(1)}, x_{N}^{(2)}, \ldots, x_{N}^{(k)}\right), N \geq 1 .
$$

Будем предполагать, что множество значений функции $f$ не содержится в одном смежном классе по любой собственной подгруппе группы $G$. Покажем, что для последовательности (11) выполнены условия теоремы 1.

Условие (5) выполняется согласно определению $\xi_{i, j}^{(N)}$.

Из соотношения (10) следует, что

$$
P^{\prime} \cdot P=\left(P_{1}^{\prime} \cdot P_{1}\right) \otimes\left(P_{2}^{\prime} \cdot P_{2}\right) \otimes \ldots \otimes\left(P_{k}^{\prime} \cdot P_{k}\right) .
$$

Для любой положительно-регулярной матрицы $P_{j}$ размера $2 \times 2$ матрица $P_{j}^{\prime} \cdot P_{j}$ неразложима (это нетрудно проверить непосредственно). С учетом (12) получаем, что матрица $P^{\prime} \cdot P$ неразложима. Наконец, в соответствии с (6) в данном случае множество

$$
\Omega=\left\{f\left(x_{1}, \ldots, x_{k}\right) \mid\left(x_{1}, \ldots, x_{k}\right) \in V_{k}\right\}
$$

не содержится в одном смежном классе группы $G$ по ее собственной подгруппе.

Таким образом, по теореме 1 и следствию 1 для последовательности (11) соответствующие последовательности (3), (4) имеют в пределе при $N \rightarrow \infty$ равномерное распределение на группе $G^{(m)}$.

Ранее данное утверждение в случае, когда $|G|=2$, было доказано И. И. Гезенко.

Вернемся к исследованию последовательностей (3), (4) для произвольной цепи Маркова (1) и рассмотрим случай, когда регулярное отображение $\varphi$ является гомоморфизмом группы $G^{(m)}$ на группу $G$. Это означает, 
что, во-первых, для некоторых эндоморфизмов $\varphi_{2}, \ldots, \varphi_{m}$ и автоморфизма $\varphi_{1}$ группы $G$ верно равенство

$$
\varphi\left(g_{1}, g_{2}, \ldots, g_{m}\right)=\varphi_{1}\left(g_{1}\right) \cdot \varphi_{2}\left(g_{2}\right) \cdot \ldots \cdot \varphi_{m}\left(g_{m}\right), g_{1}, g_{2}, \ldots, g_{m} \in G,
$$

и, во-вторых, если $m>1$, то для любого $j \in\{2, \ldots, m\}$ образ $\varphi_{j}(G)$ содержится в центре группы $G$.

Здесь мы сформулируем и докажем необходимые и достаточные условия предельной равномерности для последовательностей распределений случайных величин (4) в общем случае неразложимой цепи Маркова (1).

Введем необходимые обозначения.

Отображению $\varphi$ соответствует автоморфизм $\Phi$ группы $G^{(m)}$, определяемый соотношением

$$
\Phi\left(g_{1}, g_{2}, \ldots, g_{m}\right)=\left(g_{2}, \ldots, g_{m}, \varphi\left(g_{1}, \ldots g_{m}\right)\right),\left(g_{1}, g_{2}, \ldots, g_{m}\right) \in G^{(m)} .
$$

Пусть $\operatorname{ord} \Phi-$ порядок автоморфизма $\Phi, d-$ число циклических классов состояний цепи Маркова (1),

$$
f=\operatorname{HOK}(d, \operatorname{ord} \Phi) \text {. }
$$

Пусть $D$ - циклический класс состояний цепи Маркова (1), такой что $D \ni 1$. Рассмотрим ориентированный граф $\Gamma$ (см. [1]) с множеством вершин $D$ и множеством ориентированных ребер $\left\{e_{i, j}^{+1}, e_{j, i}^{-1} \mid i, j \in D, p^{(f)}(i, j)>0\right\}$. Путем в $\Gamma$ назовем произвольную последовательность ориентированных ребер вида

$$
z=e_{i_{1}, i_{2}}^{\varepsilon_{1}}, e_{i_{2}, i_{3}}^{\varepsilon_{2}}, \ldots, e_{i_{k}, i_{k+1}}^{\varepsilon_{k}} .
$$

Для произвольных $i, j \in D, R \in \mathbb{Z}$ обозначим через $L_{R}(i, j)$ множество всех путей $z$ вида (13), для которых $i_{1}=i, i_{k+1}=j, \varepsilon_{1}+\varepsilon_{2}+\ldots+\varepsilon_{k}=R$. Из условия $d \mid f$ следует, что матрица $\left\|p^{(f)}(i, j)\right\|_{i, j \in D}$ положительно-регулярна. Поэтому для любых $i, j \in D, R \in \mathbb{Z}$ множество $L_{R}(i, j) \neq \oslash$.

Для любых $i, j \in\{1, \ldots, n\}, N \geq 1$, определим случайные величины со значениями в группе $G^{(m)}$ :

$$
\begin{gathered}
\bar{\xi}_{i, j}^{(N)}=\left(e_{G}, \ldots, e_{G}, \xi_{i, j}^{(N)}\right) \\
\bar{\zeta}^{(N)}=\left(\left(\ldots\left(\bar{\xi}_{\alpha_{1}, \alpha_{2}}^{(1)}\right)^{\Phi} \cdot \ldots\right)^{\Phi} \cdot \bar{\xi}_{\alpha_{N-1}, \alpha_{N}}^{(N-1)}\right)^{\Phi} \cdot \bar{\xi}_{\alpha_{N}, \alpha_{N+1}}^{(N)}, N \geq 1 .
\end{gathered}
$$


$e_{G}-$ нейтральный элемент группы $G$. Для произвольных $i, j \in\{1, \ldots, n\}$, таких что $p^{(N)}(i, j)>0$, рассмотрим случайные величины

$$
\bar{\zeta}_{i, j}^{(N)}
$$

со значениями в группе $G^{(m)}$, распределения которых совпадают с условным распределением случайной величины $\bar{\zeta}^{(N)}$ при условии $\alpha_{1}=i, \alpha_{N+1}=j$. Определим подмножества элементов группы $G^{(m)}$ :

$$
\bar{\Omega}\left(e_{i, j}^{+1}\right)=\Omega\left(\bar{\zeta}_{i, j}^{(f)}\right), \bar{\Omega}\left(e_{j, i}^{-1}\right)=\Omega\left(\bar{\zeta}_{i, j}^{(f)}\right)^{-1}, i, j \in\{1, \ldots, n\}, p^{(f)}(i, j)>0 .
$$

Пути $z$ вида (13) поставим в соответствие множество элементов группы $G^{(m)}$ :

$$
\bar{\Omega}(z)=\bar{\Omega}\left(e_{i_{1}, i_{2}}^{\varepsilon_{1}}\right) \cdot \bar{\Omega}\left(e_{i_{2}, i_{3}}^{\varepsilon_{2}}\right) \cdot \ldots \cdot \bar{\Omega}\left(e_{i_{k}, i_{k+1}}^{\varepsilon_{k}}\right) .
$$

Зафиксируем произвольно $z^{\prime} \in L_{1}(1,1)$ и $x \in \bar{\Omega}\left(z^{\prime}\right)$. Кроме того, для каждого $j \in D$ зафиксируем произвольно путь $z_{1, j} \in L_{0}(1, j)$ и элемент $\sigma_{1, j} \in \bar{\Omega}\left(z_{1, j}\right)$.

Рассмотрим множество элементов группы $G$ :

$$
\bar{\Omega}=\bigcup_{\substack{i, j=\overline{, n} \\(f)(i, j)>0}} x^{-1} \cdot \sigma_{1, i} \cdot \bar{\Omega}\left(e_{i, j}^{+1}\right) \cdot \sigma_{1, j}^{-1} .
$$

Теорема 2. Пусть $\varphi$-регулярный гомоморфизм группь $G^{(m)}$ на групny $G$, тогда для любых $i, j \in\{1, \ldots, n\}$, любого распределения случайного вектора $\left(\zeta_{1}, \ldots, \zeta_{m}\right)$ последовательность распределений случайных величин (4) имеет своим пределом при $N \rightarrow \infty$ равномерное распределение на группе $G^{(m)}$ тогда и только тогда, когда множество $\bar{\Omega}$ является системой образующих элементов группь $G^{(m)}$.

Доказательство. Случайные величины (14) не зависят от вектора $\left(\zeta_{1}, \ldots, \zeta_{m}\right)$. Так как отображение $\Phi-$ гомоморфизм, то для произвольных $i, j \in\{1, \ldots, n\}, N \geq 1$, удовлетворяющих условию $p^{(N)}(i, j)>0$, любых $g_{1}, \ldots, g_{m} \in G$ условное распределение случайной величины $\zeta_{i, j}^{(N)}$ при условии $\zeta_{1}=g_{1}, \ldots, \zeta_{m}=g_{m}$ совпадает с распределением произведения $\left(g_{1}, \ldots, g_{m}\right)^{\Phi^{N}} \cdot \bar{\zeta}_{i, j}^{(N)}$. Отсюда следует, что свойство предельной равномерности для последовательностей распределений случайных величин (4) при произвольном распределении $\left(\zeta_{1}, \ldots, \zeta_{m}\right)$ равносильно свойству предельной равномерности для последовательностей распределений случайных величин (14). 
Рассмотрим цепь Маркова $\Im^{(f)}$ с множеством состояний $D$, матрицей переходных вероятностей $\left\|p^{(f)}(i, j)\right\|_{i, j \in D}$ и некоторым положительным вектором начального распределения. Произвольной паре состояний $i, j \in D$, $p^{(f)}(i, j)>0$, поставим в соответствие случайную величину со значениями в группе $G^{(m)}$, распределение которой совпадает с распределением случайной величины $\zeta_{i, j}^{(f)}$. В 33 работы [2] были определены произведения $\eta_{i, j}^{(N)}, N \geq 1$, $i, j \in\{1, \ldots, n\}, p^{(N \cdot f)}(i, j)>0$, случайных величин со значениями в $G^{(m)}$, распределения которых определяются цепью Маркова $\Im^{(f)}$ и исходными распределениями $\bar{\zeta}_{i, j}^{(f)}, i, j \in D, p^{(f)}(i, j)>0$. Из определения этих произведений и условия $\operatorname{ord} F \mid f$ следует, что если $i, j \in\{1, \ldots, n\}, N \geq 1$ и $p^{(N \cdot f)}(i, j)>0$, то распределения случайных величин $\bar{\zeta}_{i, j}^{(N \cdot f)}$ и $\eta_{i, j}^{(N)}$ совпадают.

Пусть $i, j \in\{1, \ldots, n\}, N>f, p^{(N)}(i, j)>0$, число $t \in\{1, \ldots, f\}$ удовлетворяет условию $t \equiv N(\bmod f)$ и $s=\frac{N-t}{f}$. Тогда верно равенство

$$
\begin{gathered}
\boldsymbol{P}\left(\bar{\zeta}_{i, j}^{(N)}=\bar{g}\right)=p^{(N)}(i, j)^{-1} \cdot \sum_{E_{k} \in D} p^{(t)}(i, k) \cdot p^{(N-t)}(k, j) \times \\
\times\left[\sum_{\bar{h} \in G^{(m)}} \boldsymbol{P}\left(\bar{\zeta}_{i, k}^{(t)}=(\bar{h})^{\Phi^{-N+t}}\right) \cdot \boldsymbol{P}\left(\eta_{k, j}^{(s)}=(\bar{h})^{-1} \cdot \bar{g}\right)\right], \bar{g} \in G^{(m)} .
\end{gathered}
$$

Из данного соотношения следует, что свойство предельной равномерности последовательностей распределений случайных величин (14) на группе $G^{(m)}$ эквивалентно аналогичному свойству для случайных величин $\eta_{i, j}^{(N)}, i, j \in$ $\in\{1, \ldots, n\}, N \geq 1, p^{(N \cdot f)}(i, j)>0$. Но тогда утверждение доказываемой теоремы есть следствие теоремы работы [5].

Следствие 2. Пусть $\varphi-$ регулярный гомоморфизм группь $G^{(m)}$ на группу $G$ и множество $\bar{\Omega}$ является системой образующих элементов груп${ }_{n ы l} G^{(m)}$. Тогда для любого распределения случайной величины $\left(\zeta_{1}, \zeta_{2}, \ldots, \zeta_{m}\right)$ последовательность распределений случайных величин (3) имеет своим пределом при $N \rightarrow \infty$ равномерное распределение на группе $G^{(m)}$.

Ранее утверждение, аналогичное теореме 2, но в терминах циклов цепи Маркова $\Im^{(f)}$ (см. доказательство), для случая абелевой группы $G$ и положительно-регулярной цепи Маркова (1) было доказано С. В. Глуховым.

\section{Список литературы}

1. Бахтурин Ю. А. Основные структуры современной алгебры. - М.: Наука, 1990, $320 \mathrm{c}$. 
2. Горчинский Ю.Н., Круглов И. А., Капитонов В. М. Вопросы теории распределений на конечных группах. - В сб.: Труды по дискретной математике. Т 1. - М.: ТВП, 1997, с. 85-112.

3. Егоров Б.А., Максимов Ю.И. Об одной последовательности случайных величин, принимающих значения из компактной коммутативной группы. - Теория вероятн. примен., 1968, т. 13, в. 4, с. 621-630.

4. Капитонов В.М. О скорости сходимости последовательности распределений, определяемых схемой авторегрессии на компактной группе. - Теория вероятн. примен., 1973, т. 18, в. 3, с. 608-615.

5. Круглов И.А. Принцип сходимости Клосса для произведений случайных величин со значениями в компактной группе, распределения которых определяются цепью Маркова. - Дискрет. матем., 2008, т. 20, в. 1, с. 38-51.

6. Максимов Ю.И. О цепях Маркова, связанных с двоичными регистрами сдвига со случайными элементами. - В сб.: Труды по дискретной математике. Т. 1. М.: ТВП, 1997, с. 203-220.

7. Сарымсаков Т. А. Основы теории процессов Маркова. - М.: ГИТТЛ, 1954, 208 с. 